\title{
Uso de áreas verdes para la actividad física: análisis descriptivo en escolares chilenos durante la pandemia por COVID-19 \\ Use of green areas for physical activity: descriptive analysis in Chilean schoolchildren during the COVID -19 pandemic
}

\author{
Manuel Bello Leal, Carlos Flores Martínez, Noemi Salvador Soler \& Frano Giakoni Ramírez
} Universidad Autónoma de Chile (Chile)

\begin{abstract}
Resumen. La vegetación de los centros urbanos puede proveer abundantes beneficios en el medio ambiente y en la sociedad, en este sentido, las áreas verdes pueden ofrecer oportunidades para la práctica regular de actividad física, proporcionando beneficios para la salud física y mental en la vida de la población. Objetivo: El objetivo de esta investigación es determinar la práctica de actividad física y los motivos de la no práctica en menores de 5 a 17 años en áreas verdes de la ciudad de Temuco, Chile. Método: Enfoque cuantitativo, de corte transversal y con un diseño no experimental y alcance descriptivo. Instrumentos: Se aplicó el cuestionario sobre la práctica de actividad física en áreas verdes y los motivos de no práctica. Resultados: Si bien la mayor parte de los menores tiene áreas verdes cerca de su domicilio, la mayoría de ellos no las utiliza para la práctica de actividad física, especialmente las damas. Respecto al tipo de actividades principalmente realizadas en las áreas verdes destacan aquellas más de tipo recreativo como jugar, caminar y el uso de la bicicleta. Conclusión: Los espacios para realizar actividad física existen, pero los menores requieren de interés, motivación y principalmente de apoyo familiar para practicar actividad física en las áreas verdes, por lo que se requiere de una mayor oferta de programas deportivos y/o recreativos relacionados con la práctica de actividad física en áreas verdes.
\end{abstract}

Palabras clave: Actividad física, sedentarismo, tiempo libre, áreas verdes, recreación, apoyo familiar.

\begin{abstract}
The vegetation of urban centres can provide abundant benefits in the environment and society. In this way, green areas can offer opportunities for the regular practice of physical activity, providing benefits for physical and mental health in the life of the population. Aim: to determine the practice of physical activity and the reasons for non-practice in children aged between 5 to 17 years in green areas of Temuco, Chile. Methods: quantitative approach, cross-sectional and with a nonexperimental design and descriptive scope. Instruments: A questionnaire to measure the practice of physical activity in green areas and the reasons for non-practice was applied. Results: Although most of children have green areas near their homes, most of them do not use these areas for physical activities, with the group of girls highlighting in this respect. Regarding the type of activities mainly carried out in green areas were those of recreational nature such as playing, walking and cycling. Conclusion: Spaces for physical activity exist, but children require interest, motivation and mainly family support to practice physical activity in green areas, as well as a greater supply of sports and/or recreational programs related to the practice of physical activity in green areas.
\end{abstract}

Keywords: Physical activity, sedentary lifestyle, free time, green areas, leisure time, family support.

\section{Introducción}

La pandemia del coronavirus (COVID-19) ha afectado a millones de personas en todo el mundo (Sohrabi et al., 2020) y ha afectado a muchos más. A finales de abril de 2020, aproximadamente 1.500 millones de niños (de 5 a 12 años) y adolescentes (de 13 a 17 años) comenzaron un aprendizaje a distancia tras el cierre de las escuelas (Couzin-Frankel, Vogel \& Weiland, 2020). Los cierres de escuelas, junto con adaptaciones socioconductuales adicionales (por ejemplo, distanciamiento

Fecha recepción: 20-08-21. Fecha de aceptación: 04-11-21

Noemi Salvador Soler

noemi.salvador@uautonoma.c social, cuarentena, etc.), están impactando las actividades de estilo de vida de niños y adolescentes durante las 24 horas del día. La evidencia preliminar sugiere que las restricciones sociales necesarias para reducir la propagación del COVID-19 han aumentado la participación en el comportamiento sedentario (Margaritis et al., 2020; Vanderloo, et al., 2020), generando menores oportunidades para que los niños y adolescentes realicen actividad física (Zenic et al., 2020; Moore et al., 2020; Guerrero et al., 2020). Estos comportamientos son perjudiciales para los resultados de salud cardiometabólica y psicológica a largo plazo en la población general (Grgic et al., 2018), y es posible que dichos comportamientos se conviertan en resultados de salud deficientes a largo plazo en niños y adolescentes (Carson et al., 2016). 
Bajo la misma premisa, la falta de actividad física (AF) y los malos hábitos alimentarios, se han establecido como factores de riesgo en la aparición y desarrollo de enfermedades crónicas no transmisibles de manera exponencial en los escolares (Olavarria, Delgado-Floody \& Martínez-Salazar, 2020). De acuerdo con los datos de la Organización Mundial de la Salud (Organización Mundial de la Salud, 2020) desde 1980 la obesidad se ha duplicado en todo el mundo, llegando en el año 2014 a más de 1900 millones de adultos con sobrepeso, siendo 600 millones de éstos los que padecen de obesidad (MaloSerrano, Castillo \& Pajita, 2017). Según la OMS, con respecto a la obesidad infantil existen más de 41 millones de niños menores de 5 años con sobrepeso u obesidad a nivel mundial. En 2016 había más de 340 millones de niños y adolescentes (de 5 a 19 años) con sobrepeso u obesidad (OMS, 2020). Los elevados niveles de sedentarismo y obesidad en la etapa escolar han motivado a promover la AF orientada hacia la salud, el bienestar físico y psicológico de los estudiantes, lo que constituye uno de los retos más importantes de la escuela actual y de la educación física (Camacho-Miñanco, Fernandez \& Ramírez, 2012)

Sedentarismo en Chile

Chile, en la actualidad se encuentra en el $6^{\circ}$ lugar mundial en casos de obesidad infantil y es el primero de América Latina (Olavarria et al., 2020). La actual Encuesta Nacional sobre Actividad Física y Deporte en la población de menores entre 5 a 17 años (Ministerio del Deporte, 2019) da cuenta de altos índices de inactividad en dicha población, ya que existe un $48.4 \%$ de dicha población encuestada inactiva, y un $16.5 \%$ clasificada como activa. De este porcentaje los más altos niveles de inactividad los presentan los jóvenes entre 13 y 17 años, presentando un $60 \%$ de inactividad en adolescentes de esas edades. Por otro lado, los índices más altos de actividad lo presentan los niños de entre 10 y 12 años con un $19.8 \%$ de la población, cifra que de igual manera refleja los bajos niveles de AF que presenta la población nacional.

Según datos de la Encuesta Nacional sobre Actividad Física y Deporte del Ministerio del Deporte de Chile (MINDEP, 2019), de los menores chilenos que practican deportes o $\mathrm{AF}$ en un $39.6 \%$, prefieren realizarlo en espacios abiertos como calles, plazas, sitios eriazos, parques, etc. En este sentido, los parques verdes urbanos pueden ofrecer oportunidades para la práctica regular de AF, proporcionando beneficios en la salud física, social y afectiva de la vida de la población (Felipe, Pimenta, Duarte, Silva \& Gomes, 2019). Los espacios recreati- vos no son productos del azar sino más bien la respuesta física ante las necesidades, relaciones e interacciones sociales, económicas y ambientales, entre tantos atributos que contiene la ciudad. Por tanto, los parques urbanos activos, pasivos e interactivos son una necesidad, producto y resultado del desarrollo urbano, que permiten la planificación, implementación y desarrollo de proyectos formales e informales (Ocampo, 2008)

Pandemia por COVID-19

En este contexto, mediante Resolución Exenta $\mathrm{N}^{\circ} 200$, de 20 de marzo del 2020, el Ministerio de Salud de Chile dispuso a la comunidad una serie de medidas por el brote del virus SARS-CoV-2, entre estas, la suspensión de los eventos deportivos y AF recreativa, de forma indefinida, hasta que las condiciones epidemiológicas permitan la nulidad de esta medida. Además, se instauraron cuarentenas en las principales ciudades del país, impidiendo salir de la casa y, por lo tanto, limitando la AF al aire libre (Kalazich et al., 2020).

Un estudio realizado en México da cuenta de las principales repercusiones del confinamiento o cuarentena, la cual ha generado un aumento y/o prevalencia de patrones de conducta sedentaria. Un gran porcentaje de las personas presenta alteraciones en el sueño, falta de motivación al realizar sus actividades de la vida diaria y con tendencia al estrés. Estos resultados son relevantes ya que comprueban que el sedentarismo y aislamiento social tiene repercusiones a nivel de estado físico y emocional (Rico-Gallegos et al., 2020).

El confinamiento a causa de la pandemia ha afectado la movilidad de las personas, la que a su vez ha ido en desmedro de los ya malogrados índices de AF y elevados índices de sedentarismo de la población en todo el mundo. Datos pertenecientes a la población mundial indican disminuciones en la AF y aumento en la exposición de pantallas, mostrando una radiografía de un contexto actual globalizado y confinado (Aguilar-Farias, Verdugo-Miranda \& Cortinez-O’Ryan, 2021).

Áreas Verdes

Cada vez hay más pruebas de que la disponibilidad local de espacios verdes está relacionada con la mejora de la salud (Maas, Verheij, Groenewegen, De Vries \& Spreeuwenberg, 2006; De Vries, Verheij, Groenewegen \& Spreeuwenberg, 2003). Los entornos de vida más verdes se han asociado a una reducción de la morbilidad (Maas, Verheij, De Vries, Spreeuwenberg, Schellevis \& Groenewegen, 2009) y la mortalidad (Mitchell \& Popham, 2008) en varios países de renta alta como Inglaterra y Holanda. Los mecanismos causales que subyacen a la relación entre espacios verdes y salud si- 
guen sin estar claros, aunque de la literatura surgen tres contendientes principales: proporcionar oportunidades para la AF; aliviar el estrés y la fatiga de atención; y facilitar el contacto social (De Vries et al., 2003). Hasta la fecha, las pruebas de estos mecanismos a nivel de la población consisten en gran medida en la constatación de que los entornos verdes suelen estar relacionados con niveles de AF, estrés o contactos sociales beneficiosos para la salud, sin explorar directamente el papel que desempeñan estos factores en la relación entre los espacios verdes y la salud (Sugiyama, Leslie, Giles-Corti \& Owen, 2008). Recientemente, los investigadores han empezado a comprobar que los niveles de AF podrían explicar las relaciones entre los espacios verdes y la salud encontradas en diversos contextos (Stronegger, Titze \& Oja, 2010). En Adelaida (Australia), el aumento de los niveles de caminata (para el ocio) explicaba la relación entre la percepción de espacios verdes y la salud física, y explicaba en parte también los beneficios para la salud mental de las zonas más verdes (Sugiyama et al., 2008). Por el contrario, el uso de la bicicleta (para desplazarse al trabajo) no medió en la relación entre los espacios verdes y la mejora de la salud declarada por los propios usuarios en los Países Bajos (Maas et al., 2008), y la AF en el tiempo libre no explicó la relación entre la calidad ambiental del barrio y la salud declarada por los propios usuarios en Austria.

Las áreas verdes urbanas presentan un enfoque estructurado, integrado y metódico del manejo de árboles, plantas y cualquier otro tipo de vegetación que se encuentre en las áreas verdes, es así como, estos centros urbanos juegan un papel muy importante en el crecimiento de las ciudades principalmente por la positiva contribución y aporte que hacen al medio ambiente, así como el bienestar de todas las personas (Krishnamurthy \& Nascimento, 1998).

La vegetación de los centros urbanos puede proveer abundantes beneficios en el medio ambiente y en la sociedad como tal, por ejemplo, los biológicos, físicos y socioeconómicos consistentes en la mejora del microclima y de la condición del aire, también se puede apreciar un incremento en la salud mental y físicas de las personas, se ve favorecido el desarrollo económico, siendo así las áreas verdes indispensables en cualquier estrategia medio ambiental del crecimiento sustentable de las ciudades en América latina (Krishnamurthy \& Nascimento, 1998)

Las áreas verdes urbanas pueden presentar oportunidades para la realización parcial de AF al aire libre, ofreciendo un sinfín de beneficios para el día a día de la población, como por ejemplo salud social, física y afectiva (Kalazich et al., 2020). En este sentido es importante mencionar que, según la Encuesta Nacional de Hábitos de Actividad Física, de las personas que practican AF el $39.6 \%$ de los encuestados prefiere hacerlo en espacios abiertos como plazas o parques (Ministerio del Deporte, 2019).

De acuerdo con Cortinez-O'Ryan y Aguilar-Farias (2017) solo un 1\% de las ciudades chilenas cumplen con las recomendaciones de la OMS (9 $\mathrm{m}^{2}$ por habitante). Sin embargo, la ciudad de Temuco es privilegiada en cuanto a las áreas verdes que se encuentran en el radio urbano de la ciudad y la conservación de estas, debido entre otros factores al clima asociado a su ubicación geográfica. Según la Municipalidad de Temuco (2016), esta ciudad del sur de Chile contaría con $6.03 \mathrm{~m}^{2}$ /hab. estando así sobre el promedio nacional $\left(4.1 \mathrm{~m}^{2} / \mathrm{hab}\right.$. ). Por lo anteriormente señalado, el objetivo de esta investigación fue determinar la práctica de AF en las áreas verdes y los motivos de no práctica de menores entre 5 a 17 años de la ciudad de Temuco (Chile).

\section{Material y Método}

La presente investigación, se sustentará en un método cuantitativo, descriptivo-transversal.

\section{Muestra}

La muestra fue de tipo no probabilística y estuvo conformada por 67 apoderados de niños(as) y jóvenes (43.28\% hombres y 56.72\% mujeres) con una edad media de $11.4 \pm 4$ años pertenecientes a la ciudad de Temuco, Chile. El tipo de muestreo fue por conveniencia, dado que los sujetos de estudio fueron escogidos dentro de una población (Mendieta, 2015). Los criterios de inclusión fueron a) ser apoderados de menores de entre 5 y 7 años de taller de la ciudad de Temuco (Chile); b) aceptar su participación en el estudio enviando de forma voluntaria el cuestionario Forms (Microsoft Teams), el que debían completar de manera online; c) firmar el consentimiento informado que autoriza el uso de la información con fines científicos siguiendo lo expuesto en la Declaración de Helsinki (2008). No fue aplicado ningún criterio de exclusión, ya que no se observaron respuestas con algún tipo de incoherencia y además debían completar el cuestionario obligatoriamente para poder enviarlo.

\section{Instrumento}

El instrumento fue una encuesta autoaplicada y ba- 
sada en la Encuesta Nacional de Actividad Física y Deportes en menores de 5 a 17 años (Ministerio del Deporte, 2019), la cual estuvo compuesta por dos dimensiones: 1) Práctica de ejercicio físico en áreas verdes, tipo de actividades realizadas, tiempo destinado a las actividades y si asiste o no acompañado a las áreas verdes y 2) Motivos de no práctica de ejercicio físico en áreas verdes y bajo qué circunstancias el menor asistiría a estos lugares para realizar AF. La aplicación se llevó a cabo entre el 1 de mayo y el 30 de junio de 2021 a través de la aplicación Forms (Microsoft Teams).

La encuesta fue sometida a validación de contenido (Argibay, 2006; Escobar-Pérez y Cuervo-Martínez, 2008) y analizada semánticamente para su aplicación en diversos contextos, para reducir interpretaciones idiomáticas inapropiadas (Ortiz-Gutiérrez y CruzAvelar, 2018). El instrumento fue validado a través de juicio de 8 expertos, todos investigadores y académicos con grado de doctor y con más de 5 años de experiencia en investigación y publicaciones recientes indexadas en Scopus y WoS en la línea de la AF y la calidad de vida. Los reactivos de la encuesta fueron validados por criterio de Delphi, con tres rondas de correcciones hasta obtener su aprobación (Astigarraga, 2003), que concluyó con el desarrollo de un piloto. Desde el punto de vista ético, la encuesta contó con el consentimiento informado de los participantes y adhirió a la Declaración de Singapur (2014).

\section{Análisis estadístico}

Para responder los objetivos de la investigación, se utilizó una estadística descriptiva con base a frecuencias y porcentajes para cada una de las preguntas del cuestionario. Además, se ejecutó un análisis inferencial mediante Chi-cuadrado de Pearson considerando estadísticamente significativo la obtención de $p<.05$. Todo lo anterior se ejecutó a través de Microsoft Excel (C) y el Paquete estadístico IBM SPSS 25.0 (C).

\section{Resultados}

La Tabla 1 muestra que la mayoría de los menores (80.6\%) sí tiene áreas verdes cerca de su casa, mientras que sólo $19.4 \%$ declara no tener áreas verdes cerca de su casa. De los cuales, la mayoría de ellos (84.6\%) no utiliza las áreas verdes para realizar AF. Al discriminar por sexo, la Tabla muestra que la mayoría de las damas no las utiliza (63.2\%), mientras que en el caso de los varones la mitad de ellos sí las utiliza (51.7\%). Al comparar por edades, se puede observar que sólo el rango de los menores (entre 5 y 9 años) presenta una mayor de utilización de dichas áreas (64.7\%).

Tabla 1.

\begin{tabular}{ccccc}
\multicolumn{5}{c}{ Utilización de áreas verdes cercanas a la casa } \\
& \multicolumn{4}{c}{ Utilización } \\
& No & Sí & Total \\
\hline Áreas verdes & No & $11(84.6 \%)$ & $2(15.4 \%)$ & $13(19.4 \%)$ \\
cercanas & Sí & $27(50 \%)$ & $27(50 \%)$ & $54(80.6 \%)$ \\
\hline Sexo & Hombre & $14(48.3 \%)$ & $15(51.7 \%)$ & $29(43.3 \%)$ \\
& Mujer & $24(63.2 \%)$ & $14(36.8 \%)$ & $38(56.7 \%)$ \\
\hline \multirow{2}{*}{ Edad } & 5 y 9 & $6(35.3 \%)$ & $11(64.7 \%)$ & $17(25.4 \%)$ \\
& 10 y 12 & $16(61.6 \%)$ & $10(38.4 \%)$ & $26(38.8 \%)$ \\
& 13 y 17 & $16(66.7 \%)$ & $8(33.3 \%)$ & $24(35.8 \%)$ \\
\hline
\end{tabular}

En la Tabla 2 se puede observar que sólo dos menores que no cuentan con áreas verdes cercanas a su casa asisten a realizar AF en ellas, mientras que aquellos que sí tienen áreas verdes cercanas asisten principalmente entre tres (7) y dos días (6).

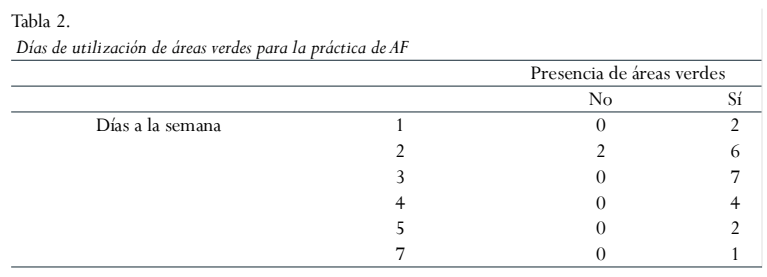

La Tabla 3 muestra que la mayoría de los menores declara asistir entre 1-2 y 3-4 días por semana a las áreas verdes para realizar AF, siendo importante resaltar que sólo fueron varones los que utilizaron entre $5 \mathrm{y}$ 7 días las áreas verdes para realizar AF.

Tabla 3.

Días de utilización de áreas verdes cercanas a la casa para la práctica

\begin{tabular}{llcccc}
\hline & & \multicolumn{3}{c}{ Días utilización } \\
& 1 y 2 & 3 y 4 & 5 y 7 & Total \\
\hline Sexo & Hombre & 5 & 3 & 3 & 11 \\
& Mujer & 5 & 8 & 0 & 13 \\
Total & 10 & 11 & 3 & 24 \\
\hline
\end{tabular}

La Figura 1 muestra que entre los menores que utilizan las áreas verdes para realizar AF, fue jugar (17) y caminar (17) las actividades que más se realizan, seguidas de andar en bicicleta (12) y practicar un deporte (9). Otros menores realizan AF en áreas verdes cuando pasean a sus mascotas (8) y otros para utilizar las máquinas de ejercicios (7) que están en los distintos puntos de la ciudad. Por último, una minoría de los menores practican otra actividad como trotar (5).

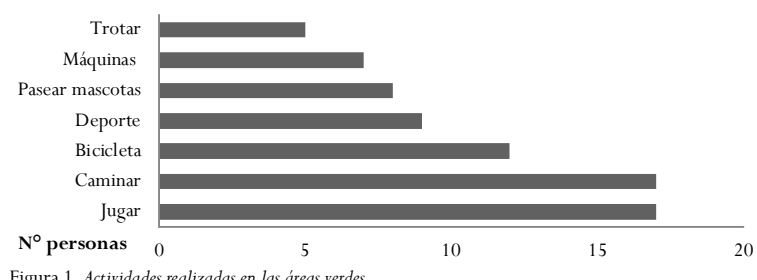

Figura 1. Actividades realizadas en las áreas verdes

El 86\% de los menores sí asisten acompañados a realizar AF a las áreas verdes (ver Tabla 4) acompañados 
principalmente de su madre (42.5\%), seguido del padre $(30 \%)$, otro familiar $(20 \%)$ y amigos $(7.5 \%)$.

\begin{tabular}{|c|c|c|c|}
\hline & pard & $\mathrm{F}$ & $\%$ \\
\hline \multirow[b]{2}{*}{ Acompañante } & No & 4 & 14 \\
\hline & Sí & 25 & 86 \\
\hline \multirow{4}{*}{ Tipo } & Madre & 17 & 42.5 \\
\hline & Padre & 12 & 30 \\
\hline & Otro familiar & 8 & 20 \\
\hline & Amigos(as) & 3 & 7.5 \\
\hline
\end{tabular}

En cuanto a las condiciones que requieren los menores para practicar algún deporte o AF, la Figura 2 muestra que la mayoría de ellos lo harían si tuviesen algún acompañante (12), seguido de si los motivaran más para la práctica de AF y deporte (8).

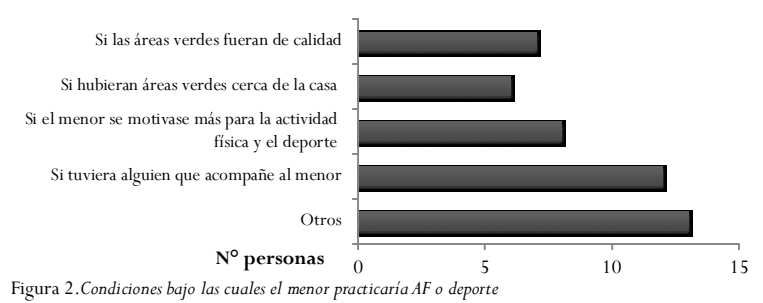

Entre las principales razones que aparecen cuando se pregunta por la no la práctica de AF y deporte, la Figura 3 muestra que esto es debido a la falta de interés (15) y al tiempo (11) encontrando una asociación estadísticamente significativa de un .000 , entre las razones y las condiciones por la práctica de $\mathrm{AF}$ en áreas verdes.

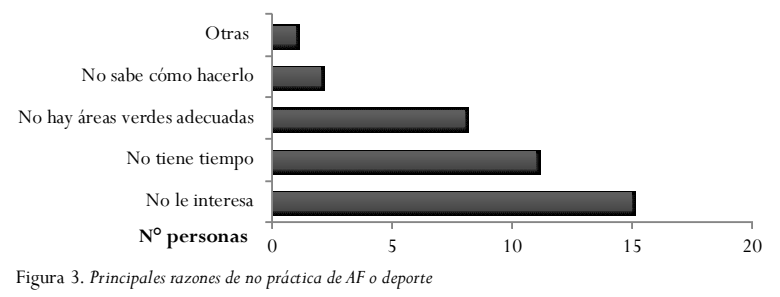

\section{Discusión}

Las áreas verdes urbanas son consideradas de gran importancia en el crecimiento de las ciudades por la positiva contribución que hacen al ambiente, así como al bienestar social, económico y principalmente en la calidad de vida de la población urbana (Flores-Xolocotzi \& González-Guillén, 2010). Los resultados de la presente investigación muestran que la mayoría de los menores cuentan con áreas verdes cerca de su casa asistiendo a ellas generalmente dos o tres días a la semana. Estos resultados serían coherentes con la mayor la disponibilidad de áreas verdes por habitante $\left(6 \mathrm{~m}^{2} / \mathrm{hab}\right.$. $)$ reportados en los documentos de diagnóstico territorial de la Municipalidad deTemuco (2016), en comparación con el promedio nacional $\left(4 \mathrm{~m}^{2} / \mathrm{hab}\right.$.). Sin embargo, y según estudios realizados por Reyes y Figueroa (2010) en otras regiones, la distribución de estas áreas verdes deja en evidencia que existe una alta brecha entre los sectores socioeconómicos más bajos y altos de la comuna, existiendo sólo dos macro-sectores de la ciudad que cumplen con las recomendaciones de la OMS ( $9 \mathrm{~m}^{2} /$ hab.), presentando en los sectores más favorecidos entre 10.4 y $14.8 \mathrm{~m}^{2}$ / hab. y en las menos favorecidas cifras inferiores a los $5 \mathrm{~m}^{2}$ por habitante. No obstante, siguen siendo superiores al promedio nacional de $2.9 \mathrm{~m}^{2} /$ hab. reportado en los municipios chilenos de menor nivel socioeconómico (Cortinez-O'Ryan \& Aguilar-Farias, 2017)

En relación con la utilización de las áreas verdes para realizar AF, los resultados de la encuesta dejan en evidencia la escasa preferencia que tienen los menores de 5 a 17 años de asistir a las áreas verdes a practicar AF, esto concuerda con la tendencia descrita previamente en la Encuesta Nacional de Actividad Física y Deportes en menores de 5 a 17 años, en la que la población inactiva físicamente alcanza el $48.4 \%$, parcialmente activos $35.1 \%$ y activos solo $16.5 \%$ (Ministerio del Deporte, 2019). Al discriminar tanto por sexo como por rango de edad, las tendencias observadas en el presente estudio concuerdan con las reportadas en la Encuesta Nacional de Actividad Física y Deportes (Ministerio del Deporte, 2019), ya que los varones son generalmente más activos al utilizar las áreas verdes para la práctica de AF (entre 5 y 7 días), coincidiendo con las observaciones de Giakoni, Paredes y Duclos (2021), presentando además una mayor utilización de las áreas verdes que las damas en esta población. Al comparar la utilización de las áreas verdes por rango de edad se observa que, al aumentar la edad, disminuye la utilización de las áreas verdes, lo que es coherente con la disminución del índice de AF al momento de la entrada en la adolescencia reportada en diversos estudios (Ministerio del Deporte, 2019; Castillo, Tornero \& García, 2018)

Por otro lado, en los menores que utilizan las áreas verdes se observa una tendencia a utilizarlas un mayor número de días cuando éstas se encuentran cercanas a su lugar de residencia, mientras que aquellos menores que no cuentan con áreas verdes cercanas escasamente se movilizan hacia ellas. En ese sentido es importante destacar como la ausencia de áreas verdes urbana se relaciona con mayor índice de marginación de acuerdo con Reyes y Gabriel (2018) y cómo precisamente con- 
tribuyen en el bienestar general especialmente en los segmentos más pobres de la población (Krishnamurthy \& Nascimento, 1998). Por otra parte, el uso cotidiano de las áreas verdes urbanas genera vínculos afectivos con el lugar, que redundan en una apropiación del lugar por parte de los individuos que utilizan las áreas verdes (Rivera, 2013).

Los encuestados que declaran realizar AF en áreas verdes, en su mayoría la utilizan para jugar y caminar, siendo esto similar a lo observado por Ugolini et al. (2020) quienes indican que las personas utilizan las áreas verdes en familia durante la pandemia para observar la naturaleza y pasear a las mascotas. En este sentido, jugar es parte fundamental del desarrollo humano, la alegría de vivir y el aprendizaje continuo (Cortinez-O'Ryan \& Aguilar-Farias, 2017; Zorrilla, 2008). Así, las áreas verdes se pueden considerar como un restaurador mental, que promueve la creatividad, siendo un tranquilizante natural y que puede coadyuvar incluso en un mejor rendimiento académico (Gareca \&Villarpando, 2017).

Es importante señalar que la realización de AF en compañía de alguien es un factor relevante a la hora de contemplar las áreas verdes como puntos idóneos para la práctica de AF (Flintoff \& Scraton, 2011). En este sentido, los resultados de la presente investigación demuestran que un $86 \%$ sí asiste acompañado a realizar AF en áreas verdes. De este alto porcentaje, destaca la importancia del núcleo familiar (la madre y el padre) como los principales acompañantes por delante de otros familiares y amigos; lo que concuerda con lo observado por Cortinez-O’Ryan y Aguilar-Farias (2017) en cuanto a que serían las madres las principales responsables de apoyar la AF y deportiva de los niños. Por otra parte, una de las razones que declararon los apoderados de los menores por la no práctica de $\mathrm{AF}$, fue el no tener un acompañante, seguido de una falta de motivación para la práctica de AF y deporte. En este sentido es importante mencionar el factor motivacional que tiene la compañía de otra persona en la práctica de AF, más aún al momento de salir de casa y dirigirse a otro espacio a realizar AF (Nuviala, Ruiz \& García, 2003).

Por otra parte, el principal motivo de no práctica declarado por los apoderados de los menores encuestados es la falta de interés, presentando gran porcentaje de respuestas identificadas con esta categoría. De acuerdo con Fuentes y Lagos (2019) y Lagos-Hernández y Fuentes-Vilugrón (2020), existirían múltiples factores que pudiesen explicar este fenómeno, tales como el cambio drástico de los intereses de los adolescentes, en el cual el uso de las tecnologías podría haber favorecido el ale- jamiento de las prácticas de AF, mermando drásticamente el interés, la motivación y el goce propio del movimiento, debido a que en los hogares es cada vez más frecuente encontrar la presencia de diversos dispositivos electrónicos (smartphones, tabletas digitales, ordenadores y videoconsolas), con lo que actualmente es denominado como «sedentarismo tecnológico», que se relaciona directamente con la obesidad (Lozano-Sánchez, Zurita-Ortega, Ubago-Jiménez, Puertas-Molero, Ramírez-Granizo \& Núñez-Quiroga, 2018). Sin embargo, los resultados de la presente investigación sugieren una asociación significativa entre las principales causas de no práctica (falta de interés) con las condiciones para la misma como la falta de acompañamiento, siendo en este caso la familia cercana lo más importante. Estos resultados serían coincidentes con lo observados por Castillo, Tornero \& García (2018) quienes destacan el tiempo compartido con la familia como un factor protector relevante frente a estilos de vida poco saludables, por lo que sería recomendable recabar más información sobre la importancia del apoyo familiar en la realización de actividades saludables como hacer AF, tal y como otros investigaciones han destacado previamente en diversas investigaciones (CortinezO’Ryan \& Aguilar-Farias, 2017; Castillo, Tornero \& García (2018).

\section{Conclusiones}

A modo de conclusión del presente estudio, se puede establecer que la no asistencia a las áreas verdes para la realización de AF se debe principalmente a la falta de interés, tornándose necesario un aumento en la motivación para asistir a las áreas verdes a realizar AF. Además, se destaca la importancia de la compañía a estas áreas principalmente de sus padres o su núcleo familiar. Además, se infiere que el no tener áreas verdes de calidad cerca de la casa del menor, podría ser una de las razones para la no asistencia a la realización de actividad física, siendo esto una muestra clara del estado y la distribución de las áreas verdes de la ciudad de Temuco.

Por otra parte, y según los resultados obtenidos, la gran mayoría de los menores asiste a estos espacios a caminar, jugar y escasamente a realizar deportes. Por tanto, se puede inferir y concluir que existe una escasa promoción de programas deportivos y/o recreativos para todo público en áreas verdes que fomenten las prácticas de AF en los menores y que ayuden a promover los hábitos de vida activa y saludable a nivel familiar.

Finalmente, los espacios para realizar actividad físi- 
ca existen, pero los menores requieren de interés, motivación y principalmente de compañía en su mayoría para practicar actividad física en las áreas verdes y lograr una disminución en el sedentarismo y un aumento en los hábitos físico-deportivos.

\section{Referencias}

Aguilar-Farias, N., Verdugo-Miranda, F. \& CortinezO’Ryan, A. (2021). Interés por la actividad física y recomendaciones recibidas con relación a conductas físicas durante etapas tempranas de la pandemia por COVID-19 en Chile. Journal of Movement and Health, 1-11. https: / / doi.org/10.5027/jmh-Vol18Issue1(2021)art115

Argibay, J. (2006). Técnicas psicométricas, cuestiones de confiabilidad y validez. Subjetividad y procesos cognitivos, 15.33. Recuperado de http:// dspace.uces.edu.ar:8180/jspui/handle/123456789/ 765

Astigarraga, E. (2003). El método Delphi. Universidad de Deusto San Sebastián. Recuperado de https:// www. codesyntax.com / prospectiva / Metodo_delphi.pdf

Camacho-Miñanco, M., Fernández, E., \& Ramírez, E. (2012). La Educación Física escolar en la promoción de la actividad física orientada a la salud en la adolescencia: una revisión sistemática de programas de intervención. Revista Complutense de Educación, 9-26. h t t p : / / d x . do i.org/ $10.5209 /$ rev_RCED.2013.v24.n1.41189

Castillo, I., Tornero, J., \& García, A. (2018). Relación entre actividad física, alimentación y familia en edad escolar. Retos: nuevas tendencias en educación física, deporte y recreación, 34, 85-88. https://doi.org/ $10.47197 /$ retos.v0i34.52782

Carson, V., Hunter, S., Kuzik, N., Gray, C., Poitras, V., Chaput, J., Saunders, T., Katzmarzyk, P., Okely, A., \& Connor, S. (2016). Systematic review of sedentary behaviour and health indicators in school-aged children and youth: An update. Applied Physiology, Nutrition, and Metabolism, 41, S240-S265. https: / / doi.org/10.1186/1479-5868-8-98

Cortinez-O Ryan, A. \& Aguilar-Farias, N. (2017). ¿Chile está comprometido con la actividad física de sus niños? Reporte de Notas chileno sobre la actividad física de niños y adolescentes 2016. Temuco, Chile. https: / / doi.org/10.13140/RG.2.2.30872.19204

Couzin-Frankel, J., Vogel, G. \&Weiland, M. (2020) School openings across globe suggest ways to keep coronavirus at bay, despite outbreaks. Science, 369, 241-245. Recuperado de: https:// www.sciencemag.org/news/2020/07/schoolopenings-across-globe-suggest-ways-keepcoronavirus-bay-despite-outbreaks

Declaración de Helsinki (2008). Principios éticos para las investigaciones médicas en seres humanos. Asociación Médica Mundial. Recuperado de: https:// www.wma.net/wp-content/uploads/2018/07/ DoH-Oct2008.pdf

Declaración de Singapur (2014). 2ndWorld Conference on Research Integrity, Recuperado de: https:// www.singaporestatement.org/Translations / SS_Spanish.pdf

De Vries, S., Verheij, R., Groenewegen, P. \& Spreeuwenberg, P. (2003). Natural environments e healthy environments? An exploratory analysis of the relationship between greenspace and health. Environment and Planning A, 35:1717e31.https: // doi.org/10.1068/a35111

Escobar-Pérez, J. \& Cuervo-Martínez, A. (2008). Validez de contenido y juicio Experto. Una aproximación a su utilización. Avances en Medición, 6, 37-36. Recuperado de: https://www.researchgate.net/ $\begin{array}{lllllllllllll}\mathrm{p} & \mathrm{u} & \mathrm{b} & \mathrm{l} & \mathrm{i} & \mathrm{c} & \mathrm{a} & \mathrm{t} & \mathrm{i} & \mathrm{o} & \mathrm{n} & /\end{array}$

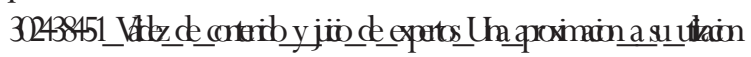

Felipe, J., Pimenta, M., Duarte, A., Silva, E. \& Gomes, G. (2019). Actividad física y ambiente: la influencia de los parques verdes urbanos en la salud. Revista Brasileira de Medicina do Esporte, 25, 4, 305-309. https: / /doi.org/10.1590/1517-869220192504187662.

Flintoff, A. \& Scraton, S. (2001). Stepping into active leisure? Young women's perceptions of active lifestyles and their experiences of school physical education. Sport Education and Society, 6(1), 5-22. https:/ / doi.org/10.1080/713696043.

Flores-Xolocotzi, R., \& González-Guillén, M. (2010). Planificación de sistemas de áreas verdes y parques públicos. Revista Mexicana de Ciencias Forestales, 1(1), 17 24. Recuperado de: https: / /www.redalyc.org/pdf/ 634/63438954003.pdf

Fuentes, G., \& Lagos, R. (2019). Motivaciones hacia la práctica de actividad física-deportiva en estudiantes de La Araucanía. Revista Ciencias de la Actividad Física UCM, 20(2). https: / / doi.org/10.29035/rcaf.20.2.3 Gareca, M., \& Villarpando, H. (2017). Impacto de las áreas verdes en el proceso de enseñanza aprendizaje. Revista Ciencia, Tecnología e Innovación, 14, 15, 877 892. Recuperado de: http: / /www.scielo.org.bo/ pdf/rcti/v14n15/v14n15_a06.pdf 
Giakoni, F., Paredes, P. \& Duclos, D. (2021). Educación

Física en Chile: tiempo de dedicación y su influencia en la condición física, composición corporal y nivel de actividad física en escolares. Retos: nuevas tendencias en educación física, deporte y recreación, 24 - 29. https://doi.org/10.47197/retos.v0i39.77781

Grgic, J., Dumuid, D., Bengoechea, E., Shrestha, N., Bauman, A., Olds, T. \& Pedisic, Z. (2018). Health outcomes associated with reallocations of time between sleep, sedentary behaviour, and physical activity: A systematic scoping review of isotemporal substitution studies. International Journal of Behavioral Nutrition and Physical Activity, 15, 69. https: / / doi.org/ 10.1186/s12966-018-0691-3

Guerrero, M., Vanderloo, L., Rhodes, R., Faulkner, G., Moore, S. \& Tremblay, M. (2020). Canadian children's and youth's adherence to the 24-h movement guidelines during the COVID-19 pandemic: A decision tree analysis. Journal of Sport Health Science, 9, 313-321. https: / / doi.org/10.1016/ j.jshs.2020.06.005.

Kalazich, C., Valderrama, P., Flández, J., Burboa, J., Humeres, D., Urbina, R. \& Smith, R. (2020). Orientaciones Deporte y COVID-19: Recomendaciones sobre el retorno a la actividad física y deportes de niños niñas y adolescentes. Revista Chilena de Pediatría, 75-90. https://doi.org/10.32641/ rchped.vi91i7.2782.

Krishnamurthy, L. \& Nascimento, J. (1998). Áreas urbanas verdes en Latinoamérica y el Caribe. Centro de agroforestación para el desarrollo sostenible, 106-361. Recuperado de: https://publications.iadb.org/es/ publicacion/15813/areas-verdes-urbanas-enlatinoamerica-y-el-caribe

Lagos-Hernández, R. \& Fuentes-Vilugrón, G. (2020). Razones para la no práctica física y deportiva en adolescentes de la región de la Araucanía, Chile. Pensar en Movimiento Revista de Ciencias del Ejercicio y la Salud, 1-14. https://doi.org/10.15517/ pensarmov.v18i2.40531

Lee, J. Mental health effects of school closures during COVID-19. (2020). Lancet Child \& Adolescent Health, 4, 421. https://doi.org/10.1016/S23524642(20)30109-7.

Lozano-Sánchez, A., Zurita-Ortega, F., Ubago-Jiménez, J., Puertas-Molero, P., Ramírez-Granizo, I., \& Núñez-Quiroga, J. (2018). Videojuegos, práctica de actividad física, obesidad y hábitos sedentarios en escolares de entre 10 y 12 años de la provincia de Granada. Retos: nuevas tendencias en educación física, de- porte y recreación, 35, 42-46. https://doi.org/ $10.47197 /$ retos.v0i35.61865

Maas, J., Verheij, R., De Vries, S., Spreeuwenberg, P., Schellevis, F. \& Groenewegen, P. (2009). Morbidity is related to a green living environment. Journal of Epidemiology Community Health, 63, 967e73. https: / / doi.org/10.1136/jech.2008.079038

Maas, J., Verheij, R., Groenewegen, P., De Vries, S., Spreeuwenberg, P. (2006). Green space, urbanity, and health: how strong is the relation? Journal of Epidemiology Community Health, 60,587e92. https: / / doi.org/ http://dx.doi.org/10.1136/ jech.2005.043125

Maas, J., Verheij, R., Spreeuwenberg, P. \& Groenewegen, P. (2008) Physical activity as a possible mechanism behind the relationship between green space and health: a multilevel analysis. BMC Public Health, 8:206. https://doi.org/0.1136/ jech. 2005.043125

Malo-Serrano, M., Castillo, N., \& Pajita, D. (2017). La obesidad en el mundo. Anales de la Facultad de medicina, 173-178. https://doi.org/10.15381/ anales.v78i2.13213

Margaritis, I., Houdart, S., El Ouadrhiri, Y., Bigard, X., Vuillemin, A. \& Duché, P. (2020). How to deal with COVID-19 epidemic-related lockdown physical inactivity and sedentary increase in youth? Adaptation of Anses' benchmarks. Archives of Public Health., 78, 1-6. https: / / doi.org/10.1186/s13690-020-00432Z

Mendieta, G. (2015). Informantes y muestreo en investigación cualitativa. Investigaciones Andina, 1148-1150. https: / / doi.org/10.33132/01248146.65

Ministerio del Deporte. (2019). Encuesta Nacional de Actividad física y Deporte en población de menores de 5 a 17 años. Gobierno de Chile. Recuperado de: https: / /sigi-s3.s3.amazonaws.com/sigi/files / 82933_encuesta_act_f_sica_y_deportes_2018_vf.pdf Mitchell R. \& Popham F. (2008). Effect of exposure to natural environment on health inequalities: an observational population study. Lancet 372:1655e60. https: / /doi.org/10.1016/S0140-6736(08)61689-X

Moore, S., Faulkner, G., Rhodes, R., Brussoni, M., Chulak-Bozzer, T., Ferguson, L., Mitra, R., O’Reilly, N., Spence, J. \& Vanderloo, L. (2020). Impact of the COVID-19 virus outbreak on movement and play behaviours of Canadian children and youth:A national survey. International Journal of Behavioral Nutrition and Physical Activity. https://doi.org/10.1186/s12966- 


\section{0-00987-8.}

Municipalidad de Temuco. (2016). Estudio actualización diagnóstico territorial para modificación al plan regulador. Recuperado de: https://www.temuco.cl/ wp-content/uploads/2018/12/Diagnostico-Territorial-2016.pdf

Nuviala, A., Ruiz, \& García, M. (2003). Tiempo libre, ocio y actividad física en los adolescentes: La influencia de los padres. Retos: nuevas tendencias en educación física, deporte y recreación, (6), 13-20. https: / / doi.org/ 10.47197/retos.v0i6.35078.

Ocampo, D. (2008). Los espacios urbanos recreativos como herramienta de productividad. Revista Escuela de Administración de Negocios, 107-125. https:// doi.org/10.21158/01208160.n63.2008.446

Olavarria, D. R., Delgado-Floody, P. \& Martínez-Salazar, C. (2020). Hábitos alimentarios, imagen corporal y condición física en escolares chilenos según niveles de actividad física y estatus corporal. Nutrición Hospitalaria, 443-449. https://doi.org/10.20960/ nh.02795

Organización Mundial de la Salud. (2020). Organización Mundial de la Salud. Recuperado de: https: / / www.who.int/es/news-room/fact-sheets/detail/ obesity-and-overweight.

Ortiz-Gutiérrez, S., \& Cruz-Avelar, A. (2018). Proceso de traducción y adaptación cultural de instrumentos de medición en salud. Actas Dermo Sifiliográficas, 103(3), 2002-2006. https://doi.org/10.1016/ j.ad.2017.09.012.

Reyes, S., \& Figueroa, A. (2010). Distribución, superficie y accesibilidad de las áreas verdes en Santiago de Chile. EURE (Santiago), 36(109), 89-110. https:// dx.doi.org/10.4067/S0250-71612010000300004

Reyes, J. \& Gabriel, C. (2018). Distribución de las áreas verdes, índice de marginación y

justicia ambiental en León, Guanajuato. Recuperado de: http: / / ru.iiec.unam.mx/3781/1/076-ReyesGabriel.pdf

Rico-Gallegos, C., Vargas-Esparza, G., PobleteValderrama, F., Carrillo-Sánchez, J., Rico-Gallegos, J., Mena-Quintana, B. (2020). Hábitos de actividad física y estado de salud durante la pandemia por COVID-19. Revista Espacios, 798-808. https:// doi.org/10.48082/espacios-a20v41n42p01

Rivera, L. (2013). Los parques urbanos como indicadores de calidad de vida, símbolos de bienestar y espacios de uso recreativo: una investigación en Bucaramanga (Colombia). Revista Universidad y Empresa, 215-237. Recuperado de: http://ri.uaemex.mx/handle/

\subsubsection{9/104605}

Sohrabi, C., Alsafi, Z., O’Neill, N., Khan, M., Kerwan, A., Al-Jabir, A., Iosifidis, C. \& Agha, R. (2020) World Health Organization declares global emergency: A review of the 2019 novel coronavirus (COVID-19). International Journal of Surgery. 76, 71-76. https:/ / doi.org/10.1016/j.ijsu.2020.02.034

Stronegger, W., Titze, S. \& Oja, P. (2010). Perceived characteristics of the neighborhood and its association with physical activity behavior and self-rated health. Health Place 16:736e43. https: / / doi.org/10.1016/ j.healthplace.2010.03.005.

Sugiyama T, Leslie E, Giles-Corti B, Owen N. (2008). Associations of neighbourhood greenness with physical and mental health: do walking, social coherence and local social interaction explain the relationships? Journal of Epidemiology \& Community Health, 62: e9. https://doi.org/10.1136/ jech.2007.064287.

Ugolini, F., Massetti, L., Calaza-Martínez, P., Cariñanos, P., Dobbs, C., Ostoiæ, S. K., Marija, A., Pearlmutter, D., Saaroni, H., Šauliene, I., Simoneti, M., Verliè, A., Vuletiæ, D., Sanesi, G. \& Sanesi, G. (2020). Effects of the COVID-19 pandemic on the use and perceptions of urban green space: An international exploratory study. Urban forestry \& urban greening, 56, 126888. https://doi.org/10.1016/ j.ufug. 2020.126888

Vanderloo, L., Carsley, S., Aglipay, M., Cost, K., Maguire, J. \& Birken, C. (2020). Applying Harm Reduction Principles to Address ScreenTime inYoung Children Amidst the COVID-19 Pandemic. Journal of Developmental Behavioral Pediatrics., 41, 335-336. https: / /doi.org/10.1097/DBP.0000000000000825.

Zenic, N., Taiar, R., Gilic, B., Blazevic, M., Maric, D., Pojskic, H. \& Sekulic, D. (2020). Levels and changes of physical activity in adolescents during the COVID19 Pandemic: Contextualizing urban vs. rural living environment. Applied Sciences, 10, 3997. https:// doi.org/10.3390/app10113997

Zorrilla, M. (2008). El juego en la infancia. Revista Chilena de Pediatría. 544-549. https: / / doi.org/10.4067/ S0370-41062008000500014. 